\title{
A new outcome measure for LUTS: Symptoms of Lower Urinary Tract Dysfunction Research Network Symptom Index-29 (LURN SI-29) questionnaire
}

\author{
David Cella PhD ${ }^{1} \mid$ Abigail R. Smith PhD $^{2} \mid$ James W. Griffith PhD \\ Kathryn E. Flynn PhD $^{3}$ | Catherine S. Bradley MD, MSCE \\ Brenda W. Gillespie PhD $^{5} \mid$ Ziya Kirkali MD ${ }^{6}$ | Pooja Talaty MS, MHA ${ }^{7}$ \\ J. Eric Jelovsek MD ${ }^{8} \mid$ Brian T. Helfand PhD $^{7} \mid$ Kevin P. Weinfurt PhD \\ the LURN Study Group \\ ${ }^{1}$ Department of Medical Social Sciences, Feinberg School of Medicine, Northwestern University, Chicago, Illinois \\ ${ }^{2}$ Arbor Research Collaborative for Health, Ann Arbor, Michigan \\ ${ }^{3}$ Medical College of Wisconsin, Milwaukee, Wisconsin \\ ${ }^{4}$ Department of Obstetrics and Gynecology, Carver College of Medicine, University of Iowa, Iowa City, Iowa \\ ${ }^{5}$ Department of Biostatistics, University of Michigan, Ann Arbor, Michigan \\ ${ }^{6}$ National Institute of Diabetes and Digestive and Kidney Diseases, Bethesda, Maryland \\ ${ }^{7}$ NorthShore University Health System, Glenview, Illinois \\ ${ }^{8}$ Department of Obstetrics and Gynecology, Duke University, Durham, North Carolina \\ ${ }^{9}$ Duke University Medical Center, Durham, North Carolina
}

\section{Correspondence}

David Cella, PhD, Department of Medical Social Sciences, Feinberg School of Medicine, Northwestern University, 633N. St Clair, 19th Floor, Chicago, IL 60611.

Email: d-cella@northwestern.edu

\section{Funding information}

National Institute of Diabetes \& Digestive

\& Kidney Diseases, Grant/Award

Numbers: DK100017, DK097780,

DK097776, DK099932, DK100011,

DK097779, DK099879, DK097772;

National Institutes of Health's National

Center for Advancing Translational

Sciences, Grant/Award Number:

UL1TR001422

\begin{abstract}
Aims: To develop a representative, self-report assessment of lower urinary tract symptoms (LUTS) for men and women, the symptoms of Lower Urinary Tract Dysfunction Research Network Symptom Index-29 (LURN SI-29).

Methods: Women and men seeking treatment for LUTS at one of six academic medical centers in the US were assessed at baseline, 3-month and 12-month intervals. Twelve-month data on 78 LURN SI-29 items were analyzed among 353 women and 420 men using exploratory factor analysis (EFA), with factor structure confirmed using confirmatory factor analysis (CFA). Internal consistency, reliability, and validity of the five developed scales were evaluated by assessing correlations with the American Urological Association Symptom Index (AUA-SI), the genitourinary pain index (GUPI), and the Pelvic Floor Distress Inventory-20 (PFDI-20), and by examining expected sex differences in scores.

Results: EFA results ( $\mathrm{n}=150$ women; 150 men) produced an interpretable eight-factor solution, with three of the factors comprised of dichotomous items addressing LUTS-associated sensations. The remaining five factors, confirmed with CFA in an independent sample of 473 participants, produced five scales: incontinence, urgency, voiding difficulty, bladder pain, and nocturia. Subscales
\end{abstract}

For a copy of the LURN SI-29, contact the corresponding author (d-cella@northwestern.edu). 
and total LURN SI-29 scores were correlated as expected with AUA-SI, GUPI, and PFDI-20. LURN SI-29 scores also performed as expected in differentiating men from women based upon clinically expected differences, with men reporting more voiding difficulties and nocturia, and women reporting more urgency and incontinence.

Conclusions: The LURN SI-29 questionnaire has the potential to improve research and clinical outcome measurement for both men and women with LUTS.

\section{K E Y W O R D S}

lower urinary tract symptoms, self-report, urinary incontinence, outcome assessment, questionnaire

\section{\begin{tabular}{l|l}
1 & INTRODUCTION
\end{tabular}}

Lower urinary tract symptoms (LUTS), common among adult men and women, ${ }^{1,2}$ can adversely affect sleep, mood, daily functioning, and work productivity. ${ }^{3-5}$ In 2012, the National Institute of Diabetes and Digestive and Kidney Diseases (NIDDK) established the symptoms of Lower Urinary Tract Dysfunction Research Network (LURN), with six tertiary care clinical research sites and a data coordinating center. The charge was to identify and explain the important subtypes of LUTS and improve the measurement of patient experiences of LUTS. ${ }^{6}$

While there are a number of validated patientreported measures of LUTS available to clinicians and researchers, no instrument captures the full spectrum of LUTS with interpretable scales that can be used for outcome measurement. For example, the LUTS tool, which captures severity and bother for a comprehensive range of symptoms, provides no scoring system nor information on important differences, making measurement of change over time challenging. The American Urological Association Symptom Index (AUA-SI) was initially developed for men with benign prostatic hyperplasia, provides scoring and interpretation information, but it does not contain questions regarding urinary incontinence (UI). Most other instruments focus on a particular symptom complex such as overactive bladder or UI but do not assess the spectrum of LUTS.

To address this gap, LURN investigators developed a Comprehensive Assessment of Self-Reported Urinary Symptoms (CASUS), ${ }^{7}$ which assesses a comprehensive set of 93 manifestations of LUTS. The goal of the current research was to reduce this comprehensive set of questions to a representative set of items for use as a clinical or research outcome questionnaire that captures aspects of LUTS relevant to both sexes. ${ }^{7}$ To develop this clinically relevant questionnaire, we used factor analyses as well as input from our large group of expert clinicians to ensure that the outcome questionnaire was psychometrically strong and included content appropriate for patients.

\section{2 | MATERIALS AND METHODS}

\section{$2.1 \quad$ Study design}

Data were obtained from the LURN Observational Cohort Study. ${ }^{8}$ Treatment-seeking men and women were recruited between June 2015 and January 2017 and completed in-person clinic visits at baseline, 3 and 12 months. At baseline, participants completed a physical examination and questionnaires related to LUTS and other symptoms. Questionnaires were repeated at 3 and 12 months. As LURN CASUS was developed while the Observational Cohort Study was underway, only a subset of participants was administered the full questionnaire at baseline ( $n=64$, women and $n=212$, men). However, most were administered LURN CASUS at 12 months. This analysis was performed using LURN CASUS responses from 12-month questionnaires, and thus included all participants that had sufficiently complete 12-month LURN CASUS forms (defined as at least $85 \%$ of the form completed).

\section{2 | Exploratory factor analyses}

Candidate subscales were identified using exploratory factor analyses (EFAs) based on a random sample of 300 participants (150 women and 150 men). Sex-specific questions, questions that were suppressed by the survey branching logic, and questions with a very low frequency of endorsement were excluded from the analyses, leaving 78 of 93 possible questions available for EFA. Because the response options included dichotomous and polytomous items, we performed multiple EFAs, specifying different factor solutions, using polychoric correlations to estimate correlations between the underlying continuous variables. Oblique rotation accounted for the known correlations between factors. For each factor identified, loadings from the individual items were provided as standardized regression coefficients (unlike correlation coefficients, standardized regression coefficients can occasionally 
exceed 1.0). Scree plots and parallel analysis were used to guide the selection of the number of useful factors. Solutions ranging from 4 to 10 factors were reviewed by eight members of the study team, and the final solution was chosen by consensus, based on the interpretability. Factor loadings greater than 0.4 were required for inclusion of an item in a designated factor. After the most interpretable factor solution was derived, the internal consistency reliability of each scale was assessed using Cronbach's alpha.

\subsection{Scale development}

Once the optimal number of factors was chosen, each factor was reviewed by the study team (two urologists, two urogynecologists, one statistician, and three outcomes researchers) who nominated the most clinically relevant items from among those with loadings above 0.4 . This process resulted in excluding three factors due to poor interpretability. All remaining factors were reviewed with relevant data, including item content and internal consistency of various item combinations, and the group reached consensus on the final scale composition of the questionnaire. This 29-item questionnaire is referred to as the LURN Symptom Index-29 (LURN SI-29).

\section{4 | Confirmatory factor analysis}

LURN SI-29 response data from a second unique sample of 471 participants ( 203 women; 268 men) with 12-month data were analyzed to assess the consistency of factor loadings with the EFA results and to evaluate the fit of the response data to the factor solution selected from the EFA process. Confirmatory factor analysis (CFA) was conducted separately for men and women. The fit was assessed using root mean squared error of approximation (RMSEA), comparative fit index (CFI), and the nonnormed fit index (NNFI).

\subsection{Scaling and initial validation}

After establishing interpretable subscales of the LURN SI29 , summed raw scores were transformed to 0-100 scale using linear transformations of each subscale raw sum. A total LURN SI-29 score was computed as the simple raw sum of all responses, also transformed to a 0-100 scale.

\section{6 | Associations between LURN SI-29 scales and validated measures of urinary symptoms}

Validity testing for the LURN SI-29 scores was performed in the LURN study population with complete 12-month
LURN SI-29 questionnaires. Initial validation of the LURN SI-29 was performed by studying its associations with concurrently administrated questionnaires that capture a wide range of symptoms experienced in people with LUTS, including the AUA-SI, ${ }^{9}$ the genitourinary pain index (GUPI) ${ }^{10}$ the pelvic floor distress inventory (PFDI-20) (administered in women only), ${ }^{11}$ and a UI severity score calculated from the LUTS tool. ${ }^{12}$ Multivariable linear regression was used to test for associations between the LURN SI-29 scales and the relevant subscales of AUA-SI, GUPI, UI severity score from the LUTS tool, and the PFDI-20 (women only). Models were run separately for men and women and both standardized and unstandardized regression coefficients were reported. All analyses were conducted using SAS version 9.4 (SAS Institute, Cary, NC).

\section{3 | RESULTS}

\section{1 | Demographic characteristics}

Table 1 provides demographic and clinical information for the EFA and CFA samples. Participants in both cohorts were on average 60 years old, mostly nonHispanic whites, and held an associate's degree or higher. The EFA sample was selected to be $50 \%$ female, whereas the CFA sample, unselected for sex, including $43 \%$ women. Most participants had AUA-SI scores in the moderate range.

\section{2 | EFA and scaling}

From the 78 candidate items, the eight-factor solution provided the most interpretable result (Table S1): incontinence (16 items), pain (16 items), voiding difficulties (12 items), urgency and bother (9 items), nighttime symptoms (3 items), sensations when needing to urinate (4 items), sensations between urinations (8 items), and sensations "that could not be put into words" ( 2 items). The last three (sensation) factors reflected the LURN effort to characterize, with dichotomous (yes/no) questions, the sensory variability of LUTS for possible diagnostic or phenotyping purposes; as such they were excluded from consideration for the LURN SI-29.

On the basis of consensus, a 6-item incontinence scale was selected (Table S2), including items on leaking at night, completely losing bladder control, leaking with feelings of urgency, leaking when laughing, sneezing, or coughing, and leaking with physical activity. Median scores (0-100 range) were 8.3 in women and 0 in men, respectively, with an interquartile range of $0-20.8$ (women) and 0-0 (men) and a score range of 0-100 in 
TABLE 1 Demographics and baseline data on participants with 12-mo LURN SI-29 completed

\begin{tabular}{|c|c|c|}
\hline & $\begin{array}{l}\text { EFA sample } \\
(n=300)\end{array}$ & $\begin{array}{l}\text { CFA sample } \\
(n=473)\end{array}$ \\
\hline Age mean (SD) & $59.3(13.5)$ & $59.7(13.4)$ \\
\hline \multicolumn{3}{|l|}{$\operatorname{Sex} \mathrm{n}(\%)$} \\
\hline Male & $150(50 \%)$ & $270(57 \%)$ \\
\hline Female & $150(50 \%)$ & $203(43 \%)$ \\
\hline \multicolumn{3}{|l|}{ Race n (\%) } \\
\hline African American & $33(11 \%)$ & $58(12 \%)$ \\
\hline Other & $25(8 \%)$ & $40(9 \%)$ \\
\hline White & $241(81 \%)$ & $373(79 \%)$ \\
\hline \multicolumn{3}{|l|}{ Ethnicity n (\%) } \\
\hline Hispanic/Latino & $17(6 \%)$ & $14(3 \%)$ \\
\hline $\begin{array}{l}\text { Non-Hispanic/non- } \\
\text { Latino }\end{array}$ & $277(92 \%)$ & $448(95 \%)$ \\
\hline Ethnicity unknown & $6(2 \%)$ & $11(2 \%)$ \\
\hline \multicolumn{3}{|l|}{ Education $\mathrm{n}(\%)$} \\
\hline$<$ High school/GED & $5(2 \%)$ & $11(2 \%)$ \\
\hline HS diploma/GED & $24(8 \%)$ & $39(8 \%)$ \\
\hline Some college & $61(21 \%)$ & $105(23 \%)$ \\
\hline Associate's degree & $25(9 \%)$ & $36(8 \%)$ \\
\hline Bachelor's degree & $87(30 \%)$ & $125(27 \%)$ \\
\hline Graduate degree & $91(31 \%)$ & $146(32 \%)$ \\
\hline \multicolumn{3}{|l|}{ Employment status n (\%) } \\
\hline Used part-time & $40(13 \%)$ & $49(11 \%)$ \\
\hline Used full-time & $115(39 \%)$ & $179(38 \%)$ \\
\hline Unemployed & $9(3 \%)$ & $16(3 \%)$ \\
\hline Not used & $133(45 \%)$ & $222(48 \%)$ \\
\hline \multicolumn{3}{|l|}{ Marital status n (\%) } \\
\hline Married/civil union & $193(65 \%)$ & $317(68 \%)$ \\
\hline Living with partner & $6(2 \%)$ & $17(4 \%)$ \\
\hline Separated/divorced & $46(15 \%)$ & $51(11 \%)$ \\
\hline Widowed & $17(6 \%)$ & $15(3 \%)$ \\
\hline Single & $37(12 \%)$ & $68(15 \%)$ \\
\hline BMI (median, IQR) & $28.9(25.7-32.9)$ & $28.6(25.1-33.1)$ \\
\hline Current tobacco user $\mathrm{n}(\%)$ & $24(8 \%)$ & $26(5 \%)$ \\
\hline Diabetes n (\%) & $46(15 \%)$ & $73(15 \%)$ \\
\hline $\begin{array}{l}\text { Functional comorbidity } \\
\text { index (median, IQR) }\end{array}$ & $2.0(1.0-4.0)$ & $2.0(1.0-3.0)$ \\
\hline PVR (median, IQR), mL & $29.5(10.0-80.0)$ & $24.0(0.0-65.0)$ \\
\hline AUA-SI (median, IQR) & $13.0(8.0-18.0)$ & $12.0(8.0-17.0)$ \\
\hline AUA QOL (median, IQR) & $4.0(3.0-5.0)$ & $4.0(3.0-5.0)$ \\
\hline
\end{tabular}

Abbreviations: AUA-SI, American Urological Association Symptom Index; BMI, body mass index; CFA, confirmatory factor analysis; EFA, exploratory factor analysis; GED, general educational diploma; IQR, interquartile range; LURN SI-29, Lower Urinary Tract Dysfunction Research Network Symptom Index-29; PVR, postvoid residual; QoL, quality of life. women and 0-45.8 in men (Figure 1A). The very low scores among men is consistent with previous descriptions of this study population. ${ }^{8}$

The 4-item bladder pain factor queries the nature, frequency, and intensity of pain and discomfort at different points in the bladder filling and emptying process. The study team review preferred frequencyover intensity-type questions; therefore the final bladder pain scale included items related to the frequency of pain and discomfort while the bladder was filling, when it was full, during urination, and right after urination (Table S2). The median rescaled score of this scale was 6.25 (interquartile range $[\mathrm{IQR}]=0-12.5$ ) in both men and women with no participant scoring the maximum possible score of 100 (maximum observed score $=75.0$ in women and 81.3 in men, Figure 1B).

After the study team presented two options for the voiding difficulty scale, the LURN Steering Committee approved an alternative 5-item version (Table S2). It consists of items related to straining, hesitancy, intermittency, weak stream, and postvoid dribble. The median score for the voiding difficulty scale was higher in men than women $(25[\mathrm{IQR}=10-40]$ vs 10 [IQR $=5-20]$ ) with scores varying across the full range of the scale in men but not in women (maximum score $=83.3$; Figure $1 \mathrm{C}$ ).

The 3-item urgency scale (Table S2) assesses how often respondents felt a sudden need to urinate, difficulty in delaying urination, and fear of leaking urine due to a sudden need to rush to urinate. The median score was $33.3(\mathrm{IQR}=16.7-50.0)$ in women and $25(\mathrm{IQR}=8.33$ 33.3) in men with scores across the full range of the scale (Figure 1D). A correlated "bother" item was retained by the Steering Committee for clinical relevance but not scaled with urgency.

A 3-item Nocturia scale (Cronbach's alpha $=0.76$ ) originally included the nighttime leakage question; however, the LURN Steering Committee felt it was more clinically appropriate in the incontinence scale and also removed the item related to nighttime urgency from the scale. Thus, the final Nocturia scale (Table S2) included items related to the number of nighttime voids and frequency of waking up due to a need to void. Scores were higher in men compared with women (median score $=71.4[\mathrm{IQR}=42.9-85.7]$ vs $57.1[\mathrm{IQR}=28.6-71.4]$; Figure 1E).

Table S3 reports inter-subscale correlations. Most of the correlations are below 0.40, indicating that each subscale is measuring a unique symptom domain. The only correlation that consistently exceeded 0.40 was that between urgency and incontinence. Urgency and pain were correlated 0.42 in women but did not exceed 0.40 in men or overall. 


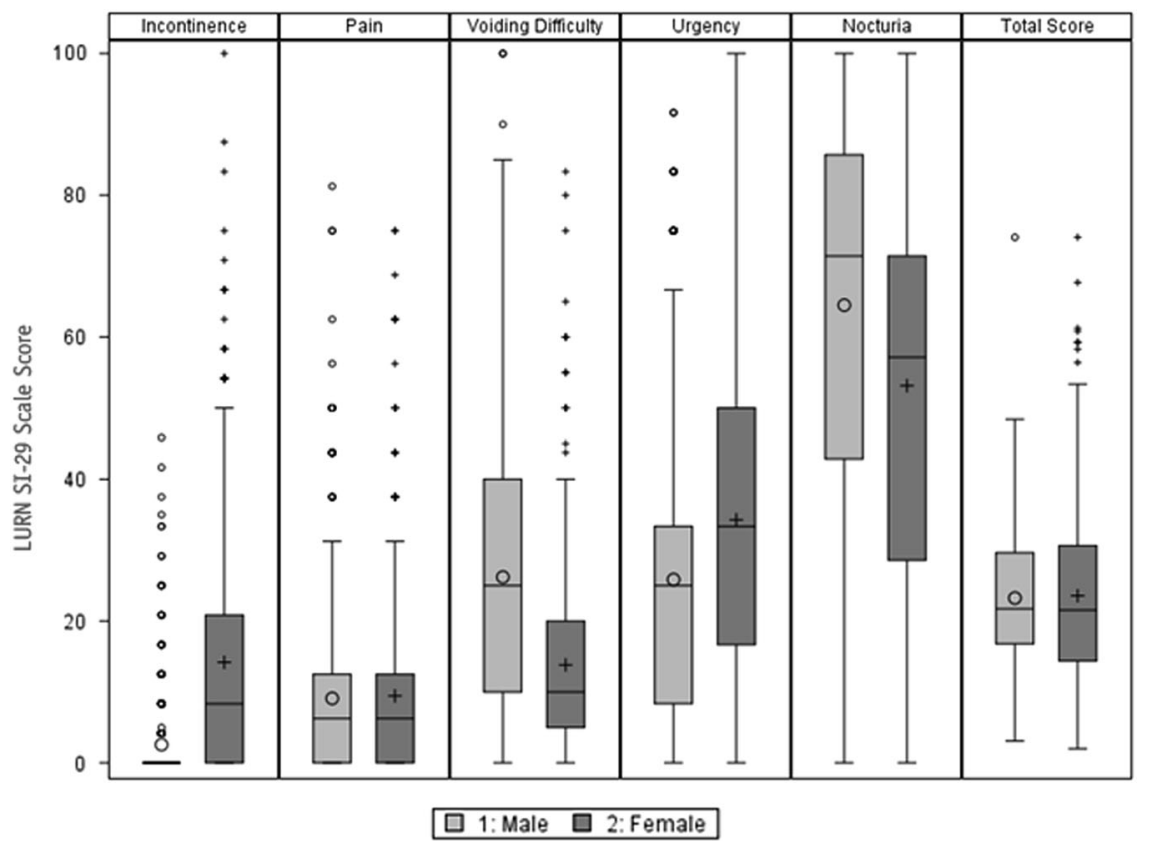

F I G U R E 1 LURN SI-29 Scales by sex ( $\mathrm{n}=353$, females and 420, males). Large circles are male means and small circles are male outliers (ie, greater than 1.5 times the interquartile range). Large pluses are female means, and small pluses are female outliers. The line in the box indicates the median, and the lower and upper edges of the box represent the 25th and 75th percentiles, respectively. The whiskers extend to 1.5 times the interquartile range (ie, the distance between the 25th and 75th percentiles). LURN SI-29, Lower Urinary Tract Dysfunction Research Network Symptom Index-29

\section{3 | Confirmatory factor analysis}

CFA showed that, as with the EFA, all factor loadings remained above 0.4 when examining a second independent dataset. Inspection of fit indices for the five-factor solution indicated a good fit based on RMSEA (Table S4). CFI and NNFI, however, ranged from 0.813 to 0.891 , suggesting that the data deviate from the hypothesized model, perhaps due to some content diversity in the correlated items comprising the scales.

\section{4 | Additional LURN SI-29 items}

The LURN Steering Committee proposed a set of remaining questions, not associated with any of the five factors, to be included on the outcome measure separately from the scales. This list comprised nine items that were deemed clinically relevant: frequency of daily voiding, nighttime urgency, constant urgency, feeling of incomplete emptying, leakage just after voiding, sex-specific questions related to splitting, spraying, or change of direction of urine stream, and overall bother. These questions were not scaled, however, they were included in a total LURN SI-29 score obtained by summing all 29 questions and rescaling from 0 to 100 .

\section{5 | Sex differences}

Figure 1 provides LURN SI-29 subscale scores separately by sex, indicating that whereas men and women report similar pain scores, women report more urgency and incontinence, and men report more voiding difficulty and nocturia. Overall, it appears that the average total LUTS burden is comparable for men and women (LURN SI-29 total median score $=21.5$ for women, 21.7 for men, see Figure $1 \mathrm{~F}$ ), but the variability of scores was significantly greater for women than for men (variance $156.62 \mathrm{vs}$ $86.00, P<.001)$.

\section{6 | Initial validation}

Table 2 demonstrates associations between LURN SI-29 scores and concurrently administered questionnaires. Multivariable models regressing the AUA-SI on the LURN SI-29 scales showed associations between the AUA-SI and all subscales in women and all subscales except incontinence in men (Table 2). The GUPI was similarly associated with all subscales in men and all subscales except nocturia in women. For men and women, the UI severity score from the LUTS tool was associated with the incontinence, voiding difficulty, and urgency scales, but not the bladder pain or nocturia scales. In women, the PFDI-20 was associated with all 


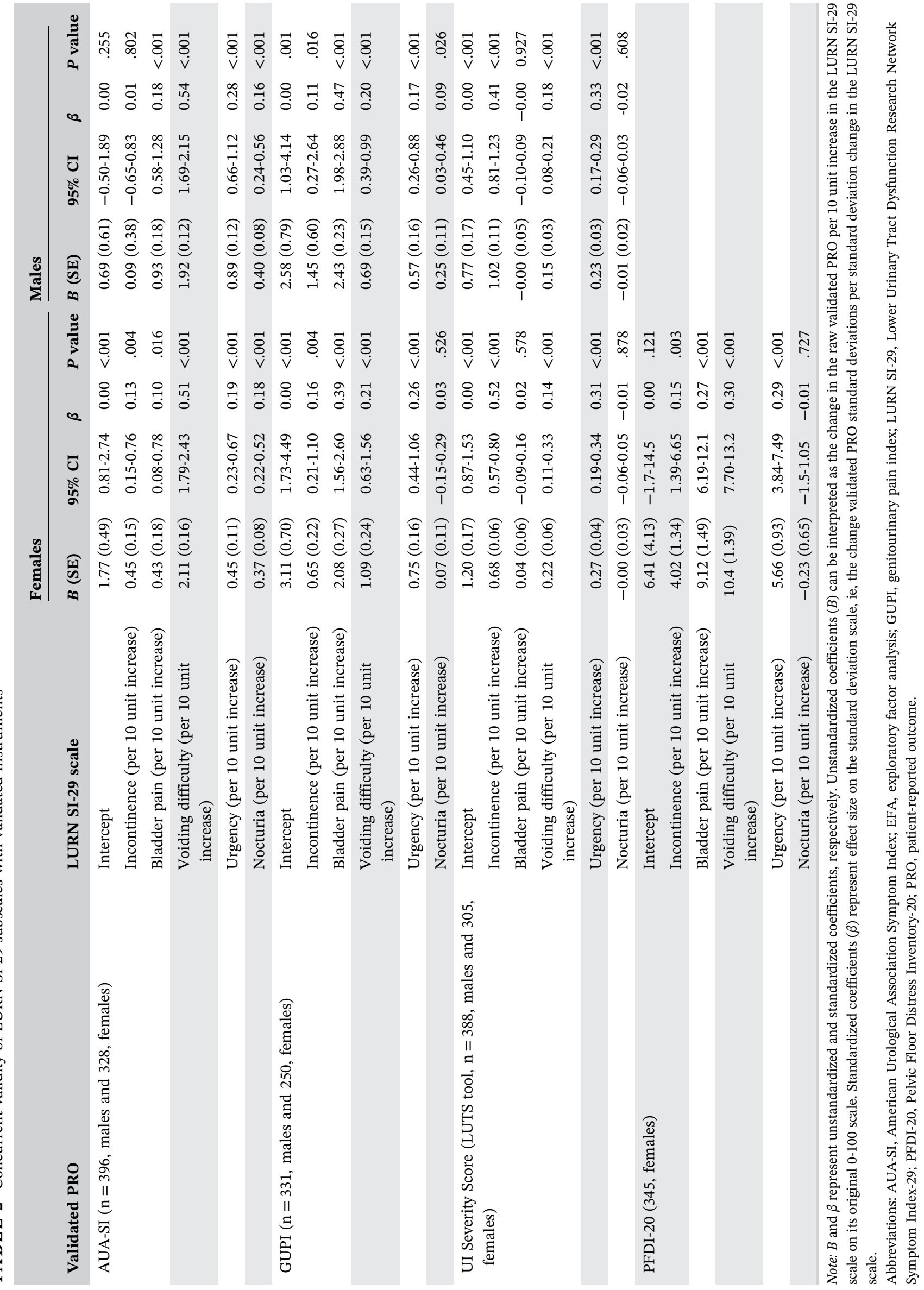


LURN SI-29 scales except nocturia. All associations were positive, that is, higher (more severe) scores on the LURN SI-29 scales were associated with higher (more severe) scores on the validated scales. When the distribution of the LURN SI-29 scales was considered by AUA-SI severity category (mild [1-7], moderate [8-19], and severe [20-35]), all scales showed an increasing trend by severity category (Figure 2), with the exception of incontinence in men, which is not measured by AUA-SI.

\section{DISCUSSION}

The goal of this research was to create an outcome measure for clinical research to quantify symptom severity across various types of LUTS. Empirically, we found that items grouped together in a clinically meaningful way, and factor loadings from EFA were replicated in an independent CFA sample. Across the total sample, all of the scales had sufficient internal consistency. Some of the CFA fit indices suggested a mismatch between the observed data and the various factor models. This is most likely due to the unique information captured by certain items within subscales (eg, for bladder pain, there are questions about pain during urination vs pain between voids). EFA and CFA served as useful guides to scale construction in this study; candidate items selected from EFA were then selected based on clinical relevance, with a specific eye toward avoiding repetition when selecting from among the items loading $>0.4$ on the respective factor. This may have had an adverse effect on the CFA fit statistics. Nevertheless, the internal consistencies of all scales remained above 0.7 in an independent sample. Thus, it may be helpful for clinicians to consider each item independently when assessing patients, in addition to minding summary scores. The converting of each scale to a 0-100 metric can enable easy comparing across scales and can aid in clinical interpretation. ${ }^{13}$

Not surprisingly, comparing men and women on responses to the LURN SI-29, women reported more urgency and incontinence, whereas men reported more voiding difficulty and nocturia. There were no sex differences in pain, nor were there differences by sex in the LURN SI-29 Total. Subscales of the LURN SI-29 were associated with relevant scales from the LUTS tool, AUA-

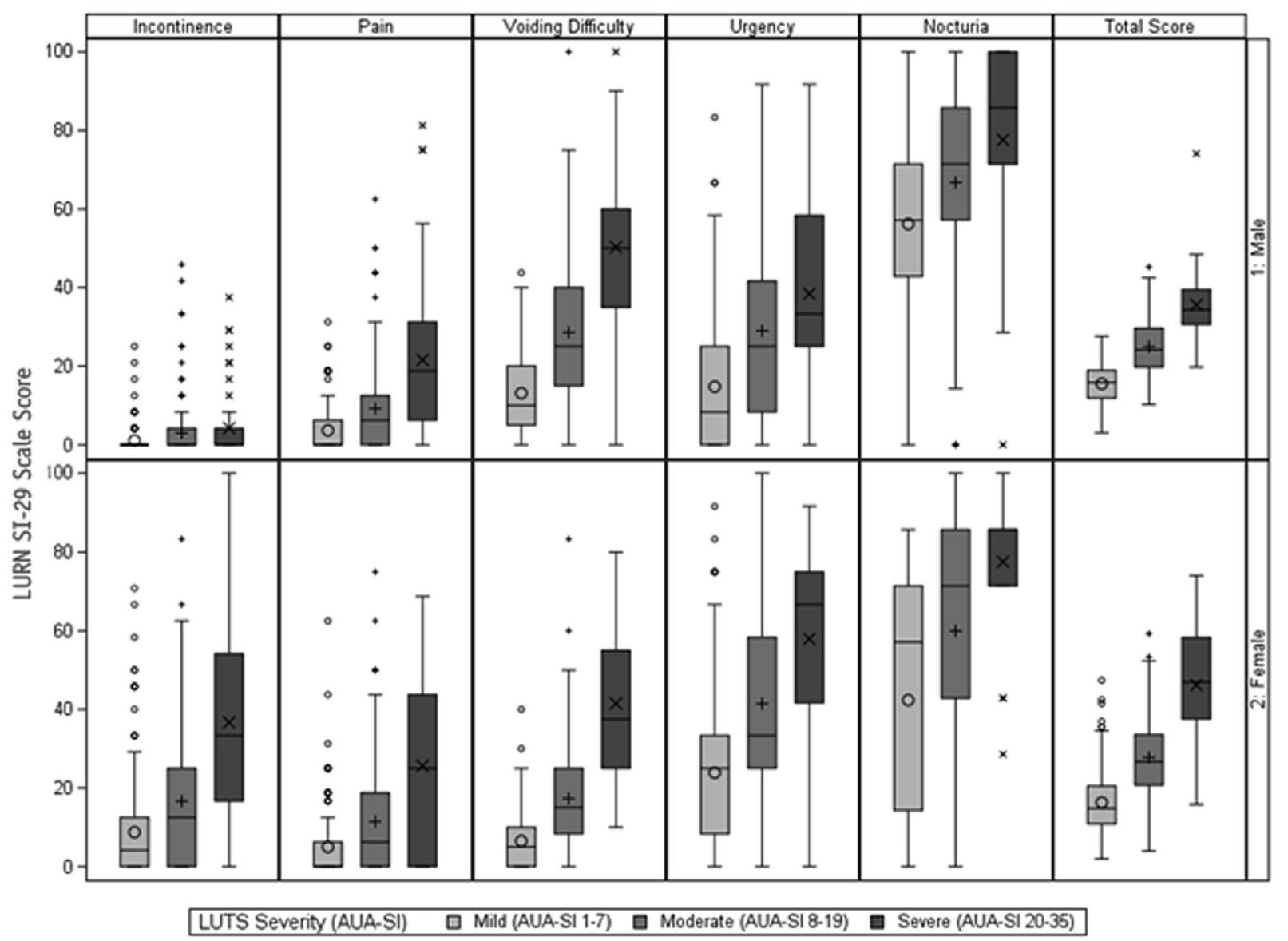

F I G U R E 2 LURN SI-29 scales by sex and LUTS severity as measured by AUA-SI severity categories (n = 335, females and 399, males). AUA-SI scores missing for $\mathrm{n}=18$ females and $\mathrm{n}=21$ males. AUA-SI, American Urological Association Symptom Index; LUTS, lower urinary tract symptoms 
SI, GUPI, and PFDI-20. These high associations with a diverse set of questionnaires offer hope that the LURN SI29 might have value across different populations, including men and women. Broadly, the data provide support for the LURN SI-29 as a potential outcome tool, so future studies should investigate its feasibility as an endpoint in clinical trials, as well as determine clinically meaningful differences between distinct patient groups, and within patient groups over time.

Strengths of our research include the multicenter study population of treatment-seeking patients with LUTS and our multidisciplinary (including input from clinicians, social scientists, and psychometricians) and a rigorous approach to measure development and testing. We also acknowledge the limitations. Our sample was not ethnically diverse and was also highly educated, and although our multicenter research enhances generalizability to other treatment-seeking populations, we have not tested the LURN SI-29 in nontreatment-seeking people with LUTS. LURN SI-29 use should be limited to patient populations pending further testing. Finally, data were not available to calculate test-retest reliability. This will be important to establish in future research.

\section{5 | CONCLUSIONS}

In summary, the LURN SI-29 is a new 29-item patientreported outcome tool. It was developed from a longer, comprehensive set of urinary symptom items developed by the LURN for use in phenotyping research. ${ }^{7}$ Five brief scales measuring urgency, incontinence, voiding difficulty, nocturia, and pain are supplemented with nine individual questions measuring voiding, nighttime urgency, constant urgency, incomplete emptying, leakage just after voiding, splitting, spraying, or change of direction of urine stream, and overall bother, together producing a total score. Internal consistency coefficients of the scales were consistently above the acceptable threshold of 0.70 , and the scales were correlated with other commonly used LUTS questionnaires. Further validation and use in LUTS outcomes research is encouraged.

\section{ACKNOWLEDGMENTS}

This study is supported by the National Institute of Diabetes \& Digestive \& Kidney Diseases through cooperative agreements (grants number: DK097780, DK097772, DK097779, DK099932, DK100011, DK100017, DK097776, DK099879). The research reported in this publication was supported at Northwestern University, in part, by the National Institutes of Health's
National Center for Advancing Translational Sciences, grant number: UL1TR001422. The content is solely the responsibility of the authors and does not necessarily represent the official views of the National Institutes of Health.

The following individuals were instrumental in the planning and conduct of this study at each of the participating institutions:

Duke University, Durham, North Carolina (DK097780): PI: Cindy Amundsen, MD, KPW, PhD; CoIs: KEF, PhD, Matthew O. Fraser, PhD, Todd Harshbarger, PhD, EJ, MD, Aaron Lentz, MD, Drew Peterson, MD, Nazema Siddiqui, MD, Alison Weidner, MD; Study Coordinators: Carrie Dombeck, MA, Robin Gilliam, MSW, Akira Hayes, Shantae McLean, MPH.

University of Iowa, Iowa city, IA (DK097772): PI: Karl Kreder, MD, MBA, CSB, MD, MSCE, Co-Is: Bradley A. Erickson, MD, MS, Susan K. Lutgendorf, PhD, Vince Magnotta, PhD, Michael A. O’Donnell, MD, Vivian Sung, MD; Study Coordinator: Ahmad Alzubaidi.

Northwestern University, Chicago, IL (DK097779): PIs: DC, BTH, MD, PhD; Co-Is: JWG, PhD, Kimberly Kenton, MD, MS, Christina Lewicky-Gaupp, MD, Todd Parrish, PhD, Jennie Yufen Chen, PhD, Margaret Mueller, MD; Study Coordinators: Sarah Buono, Maria Corona, Beatriz Menendez, Alexis Siurek, Meera Tavathia, Veronica Venezuela, Azra Muftic, Pooja Talaty, Jasmine Nero. Dr. Helfand, Ms. Talaty, and Ms. Nero are at NorthShore University HealthSystem.

University of Michigan Health System, Ann Arbor, MI (DK099932): PI: J Quentin Clemens, MD, FACS, MSCI; CoIs: Mitch Berger, MD, PhD, John DeLancey, MD, Dee Fenner, MD, Rick Harris, MD, Steve Harte, PhD, Anne P. Cameron, MD, John Wei, MD; Study Coordinators: Morgen Barroso, Linda Drnek, Greg Mowatt, Julie Tumbarello.

University of Washington, Seattle Washington (DK100011): PI: Claire Yang, MD; Co-I: John L. Gore, MD, MS; Study Coordinators: Alice Liu, MPH, Brenda Vicars, RN.

Washington University in St. Louis, St. Louis Missouri (DK100017): PI: Gerald L. Andriole, MD, H. Henry Lai; Co-I: Joshua Shimony, MD, PhD; Study Coordinators: Susan Mueller, RN, BSN, Heather Wilson, LPN, Deborah Ksiazek, BS, Aleksandra Klim, RN, MHS, CCRC.

National Institute of Diabetes and Digestive and Kidney Diseases, Division of Kidney, Urology, and Hematology, Bethesda, MD: Project Scientist: ZK, MD; Project Officer: Christopher Mullins, PhD; NIH Personnel: Tamara Bavendam, MD, Robert Star, MD.

Arbor Research Collaborative for Health, Data Coordinating Center (DK097776 and DK099879): PI: Robert Merion, MD, FACS; Co-Is: Victor Andreev, PhD, DSc, BWG, PhD, Gang Liu, PhD, Abigail Smith, PhD; Project 
Manager: Melissa Fava, MPA, PMP; clinical study Process Manager: Peg Hill-Callahan, BS, LSW; Clinical Monitor: Timothy Buck, BS, CCRP; Research Analysts: Margaret Helmuth, MA, Jon Wiseman, MS; Project Associate: Julieanne Lock, MLitt.

The protocol was approved by the institutional review boards of all participating research sites.

\section{CONFLICT OF INTERESTS}

The authors declare that there is no conflict of interests.

\section{REFERENCES}

1. Cox L, Rovner ES. Lower urinary tract symptoms in women: epidemiology, diagnosis, and management. Curr Opin Urol. 2016;26:328-333.

2. Coyne KS, Sexton CC, Thompson CL, et al. The prevalence of lower urinary tract symptoms (LUTS) in the USA, the UK and Sweden: results from the Epidemiology of LUTS (EpiLUTS) study. BJU Int. 2009;104:352-360.

3. Milsom I, Kaplan SA, Coyne KS, Sexton CC, Kopp ZS. Effect of Bothersome overactive bladder symptoms on healthrelated quality of life, anxiety, depression, and treatment seeking in the United States: results From EpiLUTS. Urology. 2012;80:90-96.

4. Vrijens D, Drossaerts J, vanKoeveringe G, VanKerrebroeck $\mathrm{P}$, vanOs J, Leue C. Affective symptoms and the overactive bladder - a systematic review. J Psychosom Res. 2015;78: 95-108.

5. Coyne KS, Wein A, Nicholson S, Kvasz M, Chen CI, Milsom I. Comorbidities and personal burden of urgency urinary incontinence: a systematic review. Int J Clin Pract. 2013;67:1015-1033.

6. Yang CC, Weinfurt KP, Merion RM, Kirkali Z LURN Study Group. Symptoms of Lower Urinary Tract Dysfunction Research Network. J Urol. 2016;196:146-152.

7. Weinfurt KP, Griffith JW, Flynn KE, et al. LURN Study Group. The Comprehensive Assessment of Self-Reported Urinary Symptoms (CASUS): A new tool for research on subtypes of patients with lower urinary tract symptoms. J Urol. 2019;201:1177-1183. [Epub ahead of print] PMID: 30730410.
8. Cameron AP, Lewicky-Gaupp C, Smith AR, et al. Symptoms of Lower Urinary Tract Dysfunction Research Network Study Group. Baseline lower urinary tract symptoms in patients enrolled in the symptoms of Lower Urinary Tract Dysfunction Research Network (LURN): a prospective, observational cohort study. J Urol. 2018;199:1023-1031.

9. Barry MJ, Fowler FJ, Jr., O'Leary MP, et al. The American Urological Association symptom index for benign prostatic hyperplasia. The Measurement Committee of the American Urological Association. J Urol. 1992;148:1549-1557.

10. Clemens JQ, Calhoun EA, Litwin MS, et al. Urologic Pelvic Pain Collaborative Research Network. Validation of a modified National Institutes of Health chronic prostatitis symptom index to assess genitourinary pain in both men and women. Urology. 2009;74:983-987.

11. Barber MD, Walters MD, Bump RC. Short forms of two condition-specific quality-of-life questionnaires for women with pelvic floor disorders (PFDI-20 and PFIQ-7). Am J Obstet Gynecol. 2005;193:103-113.

12. Helmuth ME, Smith AR, Andreev VP, et al. Use of Euclidean length to measure urinary incontinence severity based on the lower urinary tract symptoms tool. Am J Obstet Gynecol. 2018;218:357-359.

13. Cohen P, Cohen J, Aiken LS, West SG. The problem of units and the circumstance for POMP. Multivariate Behav Res. 1999;34:315-346.

\section{SUPPORTING INFORMATION}

Additional supporting information may be found online in the Supporting Information section.

How to cite this article: Cella D, Smith AR, Griffith JW, et al. A new outcome measure for LUTS: Symptoms of Lower Urinary Tract Dysfunction Research Network Symptom Index-29 (LURN SI-29) questionnaire. Neurourology and Urodynamics. 2019;38:1751-1759.

https://doi.org/10.1002/nau.24067 